\title{
TTR
}

Traduction, terminologie, rédaction

\section{Combinatoire spécialisée : trois perspectives et des enseignements pour la terminologie}

\section{Marie-Claude L'Homme}

Volume 30, numéro 1-2, 1er semestre-2e semestre 2017

La traductologie en mouvement

Translations Studies: A Forward-Moving Discipline

URI : https://id.erudit.org/iderudit/1060025ar

DOI : https://doi.org/10.7202/1060025ar

Aller au sommaire du numéro

Éditeur(s)

Association canadienne de traductologie

ISSN

0835-8443 (imprimé)

1708-2188 (numérique)

Découvrir la revue

Citer cet article

L'Homme, M.-C. (2017). Combinatoire spécialisée : trois perspectives et des enseignements pour la terminologie. TTR, 30(1-2), 215-241.

https://doi.org/10.7202/1060025ar

\section{Résumé de l'article}

Les premiers travaux portant sur la combinatoire spécialisée remontent au milieu des années 1980 et coïncident avec le début d'une période de changements profonds survenus en terminologie, tant sur le plan théorique que méthodologique. Dans le présent article, je propose une rétrospective des idées maîtresses de ces travaux et de travaux plus récents. Je tente de montrer de quelle manière ils ont contribué à une meilleure compréhension du fonctionnement des termes, notamment de leur fonctionnement linguistique. J'ai regroupé ces travaux sous trois perspectives génériques (terminographique, textuelle et lexicographique), ce qui permet de dégager leurs apports principaux à la caractérisation de la combinatoire spécialisée ainsi qu’à la réflexion théorique en terminologie. 


\title{
Combinatoire spécialisée : trois perspectives et des enseignements pour la terminologie
}

\author{
Marie-Claude L'Homme \\ Université de Montréal
}

\begin{abstract}
Résumé
Les premiers travaux portant sur la combinatoire spécialisée remontent au milieu des années 1980 et coïncident avec le début d'une période de changements profonds survenus en terminologie, tant sur le plan théorique que méthodologique. Dans le présent article, je propose une rétrospective des idées maîtresses de ces travaux et de travaux plus récents. Je tente de montrer de quelle manière ils ont contribué à une meilleure compréhension du fonctionnement des termes, notamment de leur fonctionnement linguistique. J'ai regroupé ces travaux sous trois perspectives génériques (terminographique, textuelle et lexicographique), ce qui permet de dégager leurs apports principaux à la caractérisation de la combinatoire spécialisée ainsi qu'à la réflexion théorique en terminologie..
\end{abstract}

Mots-clés : combinatoire; terminologie; collocation; banque de terminologie; corpus spécialisé

\begin{abstract}
The first publications on specialized combinatorics date back to the 1980 s and appeared in a context in which profound changes were affecting terminology both theoretically and methodologically. This article reviews the main ideas associated with these publications and more recent ones. It attempts to show how they contributed to further our understanding of the functioning of terms, and more specifically their linguistic behavior. Ideas are grouped into three general perspectives (terminography, text-based approaches and lexicography), which allow us to highlight their main contribution to the characterization of specialized combinatorics and to the overall theoretical enrichment of terminology.
\end{abstract}

Keywords: combinatorics, terminology, collocation, term bank, specialized corpus 


\section{Introduction}

Je ne répéterai pas ici que la terminologie, depuis la fin des années 1980 et le début des années 1990, connaît de profondes transformations théoriques et méthodologiques. Cette question a été explorée abondamment par différents auteurs sous des angles descriptifs ou critiques. J'ai choisi plutôt d'aborder ces changements à la lumière d'une rétrospective des travaux portant sur un phénomène particulier, à savoir celui de la combinatoire des termes.

Un retour sur les études relatives à la combinatoire spécialisée - son analyse, son traitement et son encodage dans les ressources terminologiques - est intéressant à plus d'un titre. D'abord, l'intérêt des terminologues pour ce phénomène linguistique remonte au milieu des années 1980 (Clas, 1984; Cohen, 1986; Picht, 1987; Béjoint et Thoiron, 1989) et s'est généralisé depuis. Il s'est manifesté après celui suscité par les combinaisons lexicales de langue courante, mais coïncide avec l'apparition de techniques permettant d'accéder facilement aux contextes linguistiques dans lesquels figurent les termes (corpus textuels de format électronique et outils pour les interroger). Par ailleurs, l'étude et la description du phénomène incarnent indirectement les multiples changements théoriques et méthodologiques survenus au cours des vingt-cinq ou trente dernières années en terminologie. La prise en compte de la combinatoire des termes suppose en effet l'exploration d'une dimension linguistique. Cette exploration entraîne une déstabilisation de modèles théoriques plus «classiques» de la terminologie puisque ceux-ci s'articulent autour d'une délimitation rigoureuse de concepts, délimitation faisant abstraction des propriétés linguistiques des termes exprimant ces concepts. Elle entraîne également un aménagement des ressources terminologiques dont la structure et les méthodes de compilation sont dérivées de ces modèles. Enfin, elle met au jour le fait que, pour certains utilisateurs de ressources terminologiques, il faut davantage que des renseignements visant à expliciter la nature d'un concept et sa place dans une organisation des connaissances pour être en mesure d'utiliser un terme adéquatement dans un texte.

Après avoir caractérisé le type de combinatoire étudié en terminologie (section 2), je passerai en revue des travaux qui ont abordé le phénomène selon trois perspectives. Le découpage en trois perspectives est imparfait puisqu'il existe forcément des recoupements qu'une telle division rend moins perceptibles. Il a toutefois 
le mérite de mettre au jour l'apport de différents travaux à la compréhension de la combinatoire spécialisée. J'examinerai d'abord quelques propositions visant à rendre compte de la combinatoire dans les ressources terminologiques (section 3). Nous verrons ensuite que certains chercheurs se sont focalisés sur l'intérêt que présente l'analyse de la combinatoire dans le texte spécialisé (section 4). La section 5 aborde une méthode de traitement des termes et de leur combinatoire suivant une approche lexicographique. Dans la section 6 , je dresse un bilan de ces travaux et de leur contribution à la compréhension de la combinatoire spécialisée et à la réflexion en terminologie en général.

\section{Phénomènes de combinatoire}

Le phénomène qui nous intéresse ici peut être caractérisé sommairement par la présence d'une affinité entre certaines unités lexicales, affinité qui ne peut être expliquée uniquement par les propriétés syntaxiques ou sémantiques de ces unités. Les groupes d'unités lexicales ayant ces affinités sont généralement appelés collocations par les linguistes, les lexicologues et les lexicographes. Pour les uns, l'«affinité» se perçoit par la fréquence, la récurrence de combinaisons (Sinclair, 1991). Cette caractérisation se prête bien à des applications comme le repérage automatisé de collocations dans de grands corpus textuels. Pour d'autres, elle s'explique par des contraintes lexicales : une première unité lexicale (appelée base ou mot clé) est choisie librement par le locuteur; le choix de la seconde unité lexicale (le collocatif) est imposé par celui de la base (Haussman, 1979; Mel’čuk, 1996). Des exemples classiques sont reproduits ci-dessous :

fumeur invétéré, poser une question, amour fou (collocations à base nominale)

grièvement blessé, fort comme un tigre (collocations à base adjectivale)

boire comme un trou, avancer à pas de tortue (collocations à base verbale)

La seconde définition permet de distinguer les collocations d'autres assemblages lexicaux et de raisonner leur encodage dans les dictionnaires. Les collocations se distinguent des expressions figées en ce sens qu'elles sont partiellement ou entièrement compositionnelles. Elles ne peuvent donc pas être traitées comme des entrées dictionnairiques à part entière. Elles se distinguent toutefois des 
groupements libres en ce sens qu'elles sont imprévisibles et qu'elles doivent être apprises. Leur imprévisibilité justifie donc l'intérêt de leur recensement dans les dictionnaires. La distinction entre base (unité lexicale choisie librement) et collocatif (unité lexicale dont le choix est imposé par la base) mène vers un encodage de ces combinaisons dans l'entrée consacrée à la base. Ainsi, poser une question apparaîtra dans l'entrée consacrée à question; de même, tendre un piège sera encodé dans l'entrée consacrée à piège et ainsi de suite ${ }^{1}$. J'ai reproduit ci-dessous des exemples d'entrées apparaissant dans deux dictionnaires consacrés aux collocations.

impact I $n$. 1. to have an on, upon 2. a considerable, strong; dramatic; emotional $\sim 3$. On $\sim$ (the pole collapses on $\sim$ ) (Benson et al., 1986, p. 126)

smoker: $\underline{\mathrm{ADJ}}$. chain, heavy | regular | light | passive the risk of lung cancer in passive smokers | cigar, cigarette, pipe

\section{SMOKER + NOUN a smoker's cough}

(Oxford Online Collocation Dictionary, 2018)

La caractérisation qui précède s'applique, on l'aura compris, aux combinaisons observées en langue courante. Toutefois, les terminologues ont tôt fait de la transposer aux phénomènes d'affinité entre termes et autres unités lexicales, avec des conséquences plus ou moins grandes pour les principes théoriques de la discipline et, forcément, pour les principes méthodologiques dérivés de la théorie. J'aurai l'occasion de revenir sur certaines de ces conséquences. Il suffit de dire pour l'instant qu'une différence essentielle entre les groupements décrits en lexicographie et ceux pris en compte en terminologie réside dans le fait que la dernière se concentre sur les combinaisons dans lesquelles la base est un terme relié à un domaine de spécialité (Picht, 1987). Des exemples sont reproduits ci-dessous :

(Bourse) chute des importations, importations excédentaires (Cohen, 1986, p. 64)

1. Cela dit, même si on peut appliquer cette distinction lors de l'encodage de collocations, l'accès peut se faire à partir de l'une ou l'autre de leurs composantes (sur support électronique, notamment).

2. Le dictionnaire de Benson et al. décrit des collocations comme celles dont je viens de donner une caractérisation, mais aussi des groupements que les auteurs appellent collocations grammaticales qui s'éloignent de cette caractérisation. Par exemple, des groupements composés d'une unité lexicale et d'une préposition y sont répertoriés : admiration (for), acceptable (to), (by) accident (1986 : p. ix) 
(Droit) Abroger, violer, respecter une loi

(Béjoint et Thoiron, 1989, p. 668)

(Informatique) Créer un fichier

(Heid et Freibott, 1991, p. 78)

(Stockage d'information) erasable disk

(Meyer et Mackintosh, 1994, p. 5)

(Commerce) se constituer en société

(Larivière, 1998, p. 183)

Par ailleurs, comme les termes pris en compte sont généralement des noms (Béjoint et Thoiron, 1989), les collocatifs appartiendront forcément aux parties du discours du verbe, de l'adjectif ou du nom (L'Homme, 2000; L'Homme et Jia, 2015), comme l'illustrent les exemples ci-dessus.

Or, l'«affinité» entre composantes d'une collocation, si elle peut être perçue intuitivement par un locuteur natif en langue courante, est moins immédiatement perceptible en langue spécialisée, notamment pour les personnes qui n'ont pas une connaissance approfondie des usages linguistiques propres aux domaines de spécialité (comme les traducteurs, les rédacteurs techniques ou les terminologues). Cette différence explique peut-être l'hésitation des terminologues qui, même s'ils reconnaissent l'existence de certains usages préférentiels, hésitent à étiqueter les groupes comme des collocations. Certains préféreront parler de cooccurrents (Cohen, 1986; Béjoint et Thoiron, 1989; Caignon, 2001); de combinaisons lexicales spécialisées (Meynard, 1998; L'Homme, 2000) et même de phraséologismes (Pavel, 1993) 3 ou de phrasèmes (Meyer et Mackintosh, 1994), alors que d'autres s'aligneront sur le terme employé en lexicographie, à savoir collocations (Heid et Freibott, 1991; Larivière, 1998; Williams, 1998)4. Malgré ces hésitations, on remarque que les groupements ayant retenu l'attention en terminologie partagent grosso modo les traits suivants :

3. Ici, la terminologie rompt avec une tradition linguistique, où le terme phraséologisme s'utilise souvent pour étiqueter les expressions multilexémiques non compositionnelles comme les expressions figées.

4. La liste donnée dans le texte renferme les expressions utilisées pour étiqueter les groupements en tant que tels. On trouve également des expressions pour étiqueter le phénomène selon lequel termes et unités lexicales se combinent en fonction d'usages préférentiels, par exemple, phraséologie (Picht, 1987; Pavel, 1993; Meyer et Mackintosh, 1994). 
- Ils sont composés d'un terme (unité linguistique associée à un domaine de spécialité) et d'un autre terme ou unité lexicale (de nature générale);

- Le terme est défini comme la base du groupement; l'autre terme ou unité lexicale comme le collocatif;

- Le terme est de nature nominale;

- La combinaison des deux unités est conventionnelle, c'est-àdire qu'elle correspond à un usage spécifique dans un domaine de spécialité.

Cela dit, même si la nature des groupes lexicaux retenus en terminologie fait une relative unanimité, les approches adoptées pour les décrire ne sont pas uniformes. Trois de ces approches font l'objet des sections qui suivent.

\section{La combinatoire : perspectives terminographiques}

Dès la première heure, la réflexion sur la combinatoire spécialisée a consisté à se pencher sur des manières d'intégrer des groupements plus longs que le terme dans les ressources terminologiques et plus particulièrement dans les banques de terminologie. Les utilisateurs de ces ressources, notamment les traducteurs, même s'ils y puisaient de nombreux renseignements utiles quant au concept dénoté par le terme et à sa place dans un domaine de spécialité, devaient consulter d'autres ressources pour trouver des renseignements sur l'usage des termes dans les textes (Heid et Freibott, 1991).

Les ressources terminologiques n'ont pas été conçues à l'origine pour accueillir les collocatifs de termes. L'information linguistique y est minimale (elle se limite généralement à l'indication de la partie du discours), et la prise en compte de la combinatoire spécialisée suppose un examen plus approfondi du comportement linguistique des termes. Or, la structure de ces ressources, notamment celle des banques de terminologie, est organisée autour du postulat selon lequel les termes dénotent des concepts : une entrée est donc consacrée à un concept dont la place est définie rigoureusement au sein d'un domaine de connaissances. Il s'agit peut-être là de l'une des raisons pour lesquelles l'ajout de collocatifs n'est pas une pratique généralisée dans les ressources terminologiques. Il existe toutefois quelques propositions allant de ce sens, propositions qui font l'objet des sections suivantes. 


\subsection{Ajout de collocatifs à des entrées terminologiques existantes}

Une première solution consiste à ajouter des rubriques à des fiches terminologiques existantes, fiches dont les vedettes sont des termes nominaux. C'est la solution adoptée dans Termium Plus $(2018)^{5}$, qui propose quatre rubriques consacrées à la phraséologie. Les collocatifs sont répartis en fonction de leur partie du discours et, dans le cas des collocatifs nominaux, de leur position par rapport au terme faisant l'objet de la fiche (voir la figure 1 montrant ces rubriques pour le terme logiciel). La solution a le mérite d'être simple et facile à implémenter, puisqu'elle ne change pas en profondeur la structure des fiches et nébranle pas le socle conceptuel sur lequel cette structure est érigée.

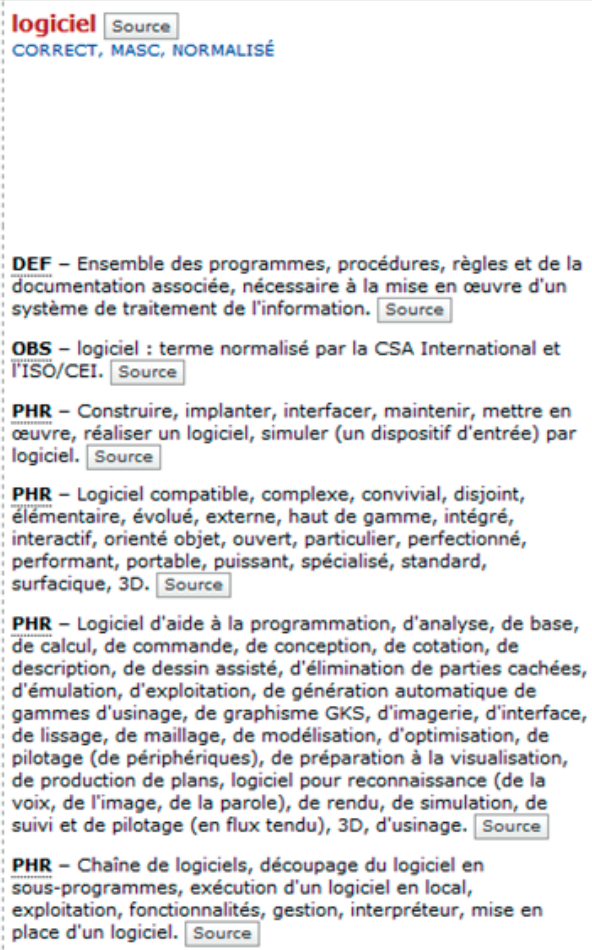

Figure 1. Fiche logiciel (Termium Plus, 2018)

5. C'est également la solution retenue dans un certain nombre de dictionnaires papier; voir notamment Lainé (1993), Maynard (1998) et Caignon (2001). 
L'intégration de collocatifs à des entrées terminologiques existantes fait émerger une première question pour la terminologie, à savoir celle de la distinction entre terme complexe et collocation. Le problème se pose pour les groupes composés d'un terme modifié par un adjectif ou un syntagme prépositionnel (Meyer et Mackintosh, 1994; L'Homme et Jia, 2015) ${ }^{6}$. Les ressources terminologiques - les banques de terminologie ne font pas exception - recensent de très nombreux termes complexes compositionnels. Ainsi, si l'on se reporte à Termium Plus, on remarque que la banque renferme des fiches consacrées à logiciel de modélisation, logiciel d'analyse, logiciel d'émulation, etc. Or, ces mêmes groupes figurent également comme "phraséologismes» dans la fiche consacrée à logiciel (figure 1). Envisagée à l'échelle d'une banque de terminologie, cette absence de distinction mène forcément à un encodage incohérent des expressions multilexémiques.

L'ajout de collocatifs à des entrées terminologiques existantes présente d'autres inconvénients méthodologiques qui ont rapidement été relevés (Béjoint et Thoiron, 1989; L'Homme, 1995). Si l’on se reporte à l'exemple de Termium Plus (2018), la banque renfermera inévitablement des fiches consacrées à des types de logiciels (logiciel d'application, logiciel de vérification, didacticiel, etc.). Or, une partie des collocatifs répertoriés pour logiciel se combinent également avec ces termes (par exemple, didacticiel convivial, logiciel d'application performant). Il faudrait donc reproduire des listes entières de collocatifs dans de nombreuses fiches, ce qui entraînerait une certaine redondance. Dans Termium Plus, les rubriques consacrées à la phraséologie n'apparaissent pas dans toutes les fiches, mais aucun mécanisme n'est prévu pour signaler que certains collocatifs se combinent avec d'autres termes décrits dans la banque. L'utilisateur devra donc avoir le réflexe de consulter une fiche consacrée à un terme simple et en déduire qu'une partie des collocatifs qui apparaissent dans sa description sont valables pour d'autres termes appartenant à la même classe conceptuelle.

Ce phénomène de combinaison des collocatifs avec des ensembles de termes a été mis en évidence par Willy Martin (1992) et Ulrich Heid (1994). Heid (1994) a proposé de distinguer, d'une

6. Le problème ne se pose pas directement pour les groupes composés d'un terme et d'un verbe, puisque les ressources terminologiques classiques répertorient peu de verbes. 
part, les collocations lexicales (où un collocatif s'observe avec un seul terme) et, d'autre part, les collocations conceptuelles (où un collocatif se combine avec des ensembles de termes appartenant à la même classe).

\subsection{Adapter les ressources terminologiques à la description de la combinatoire spécialisée}

Ulrich Heid et Gerhard Freibott (1991) ont proposé une solution différente de celle qui consiste à dresser une liste de collocatifs dans une entrée consacrée à un terme de nature nominale. Ils suggèrent de créer des entrées pour les collocations en tant que telles. Comme leur méthode est implémentée dans une base de données relationnelle, un système de pointeurs permet d'accéder aux collocations à partir de chacune de leurs composantes et, inversement, aux composantes à partir des collocations. L'implémentation permet un accès flexible aux collocations à partir de la collocation elle-même, ou encore, à partir de la base ou du collocatif. On ne sait pas, cependant, si et de quelle manière la distinction entre terme complexe et collocation est prise en charge, ni si une distinction entre collocations lexicales ou collocations conceptuelles est établie.

Au milieu des années 1990, j’ai proposé d'aller un peu plus loin dans le traitement de certains collocatifs (les collocatifs verbaux) dans les banques de terminologie et ainsi prendre en charge le problème des collocatifs se combinant avec des ensembles de termes (autrement dit, les collocations conceptuelles de Heid, 1994) (L'Homme, 1997). L'idée consiste à créer des entrées pour les verbes puisque certains verbes peuvent prétendre au statut de termes (Picht, 1987; L'Homme, 1998) - et à fournir une description détaillée de ces verbes. Ces entrées rendent compte de la structure actancielle des verbes au moyen d'un système de classes conceptuelles. Par exemple (figure 2, page suivante), en tenant pour acquis que le verbe imprimer a trois actants, ceux-ci peuvent être représentés au moyen des classes suivantes : <UTILISATEUR>, <DONNÉES> et <IMPRIMANTE>. La première classe sert à rendre compte de termes comme usager, utilisateur, informaticien, programmeur, la seconde représente des termes comme fichier, données, document, fichier exécutable; la troisième, enfin, rend compte de termes comme imprimante, imprimante laser, imprimante à jet d'encre, imprimante réseau. 


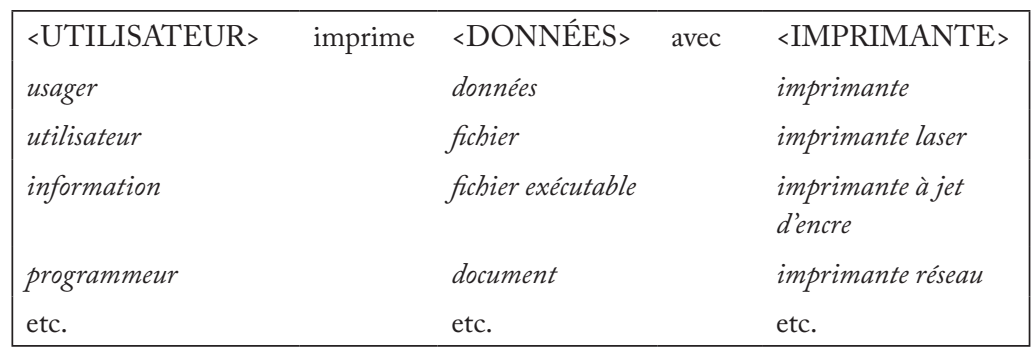

Figure 2. Représentation de la structure actancielle du verbe imprimer au moyen d'un système de classes conceptuelles

Il suffit par la suite d'indiquer la classe conceptuelle à laquelle appartiennent les termes dans leurs fiches respectives pour lier terme et collocatif. Par exemple, si imprimante laser fait partie de la classe <IMPRIMANTE>, elle peut admettre le collocatif imprimer (figure 3).

imprimante laser, n. f.

Définition : Imprimante électrophotographique permettant la reproduction de ...

Classe conceptuelle <IMPRIMANTE>

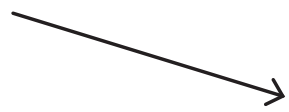

<UTILISATEUR> imprime <DONNÉES> avec <IMPRIMANTE>

Figure 3. Lien entre entrées terminologiques et structure actancielle de verbes

Les deux solutions présentées dans cette section exigent que des aménagements soient faits aux banques de terminologie (entrées consacrées aux collocations, entrées consacrées aux verbes, rubriques pour décrire les classes auxquelles appartiennent les termes). La seconde solution exige également la mise en place d'un système de classes dans chacun des domaines décrits. Cependant, comme les solutions précédentes, elles n'altèrent pas leur structure en tant que telle: elles sont superposées à une structure préexistante et ne viennent pas bouleverser des habitudes d'encodage de données terminologiques. 
De manière générale, l'ajout de collocatifs à des entrées terminologiques existantes pose la question du mélange de deux plans descriptifs. D'une part, on isole une entité appelée concept, méthode qui suppose un ancrage dans le système conceptuel du domaine de connaissances auquel il y est lié. D'autre part, on associe à cette entité des unités linguistiques, méthode qui repose sur l'observation de termes dénotant des concepts dans les textes. Il est permis de se demander si ces deux plans descriptifs sont entièrement compatibles.

\section{La combinatoire : perspectives textuelles}

Aux défis que soulève l'encodage de la combinatoire dans les ressources terminologiques s'ajoute celui de son analyse dans les corpus spécialisés. L'accès généralisé à de grands corpus textuels en format électronique et l'existence d'outils pour les interroger permettent d'obtenir des perspectives diverses sur les assemblages lexicaux. Assez tôt, les chercheurs se sont intéressés au repérage automatique ou assisté des collocations de nature générale (Church et Hanks, 1990; Smadja, 1991; Kilgarriff et Tugwell, 2001), mais ces travaux peuvent être portés au repérage de collocations spécialisées.

L'intérêt des méthodes automatiques ou semi-automatiques est indéniable. Outre le fait qu'elles forent de grands corpus rapidement et d'une manière systématique, elles mettent au jour des phénomènes que la lecture linéaire d'un texte ne permet pas de voir aussi facilement. Cependant, malgré leur intérêt, il appartient toujours à l'analyste (et donc au terminologue) de statuer sur le type de combinaisons extraites et sur leur intérêt pour l'analyse terminologique. Les deux sections suivantes présentent des pistes intéressantes pour le terminologue.

\subsection{La combinatoire comme moteur d'un réseau conceptuel}

Geoffrey Williams (1998) s'intéresse à l'exploration de la combinatoire en corpus spécialisé et à sa contribution à l'analyse conceptuelle. Selon lui, l'analyse des collocations dans lesquelles les termes ou unités lexicales apparaissent constitue un bon moyen d'identifier et de délimiter les concepts d'un domaine, puisque les concepts dénotés par les termes ne sont pas directement observables. L'auteur va plus loin et tient pour acquis que la combinatoire des termes, telle qu'observée dans les textes associés à un domaine (l'auteur s'intéresse à la biologie végétale) et à un genre textuel spécifique (les articles de recherche), permet de mettre au jour la structure conceptuelle d'une sous-langue. 


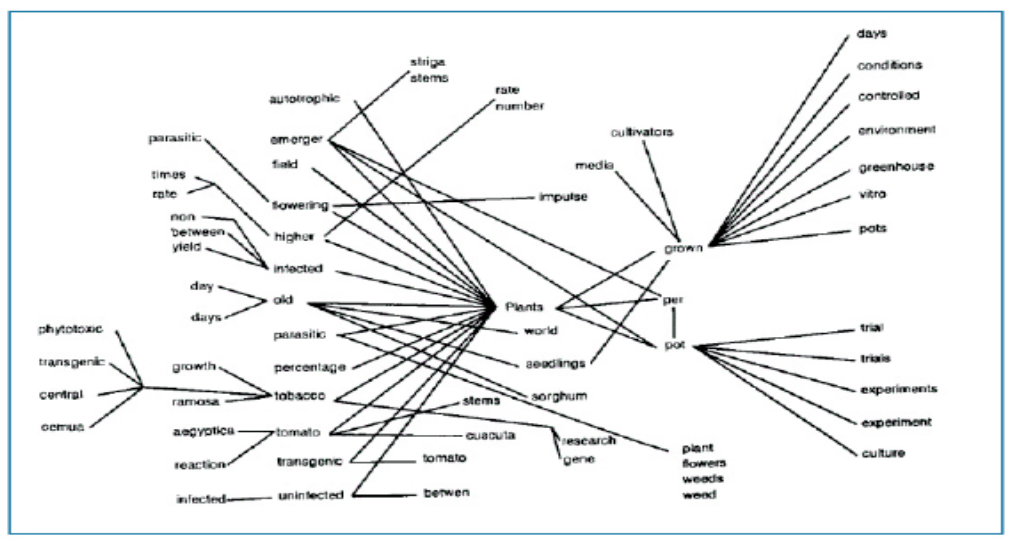

Figure 4. Réseau collocationnel ayant au centre plants (Williams, 1998, p. 169)

Williams procède par la construction d'un réseau dans lequel les nœuds correspondent aux éléments d'une collocation (des items lexicaux présents dans le corpus spécialisé). L'auteur fait appel à une mesure appelée information mutuelle pour dégager les collocations. Cette mesure a été utilisée préalablement par Ken Church et Patrick Hanks (1990) et évaluée à des fins lexicographiques ${ }^{7}$. Chacun des nœuds d'une première collocation peut mener à d'autres liens collocationnels qui, à leur tour, mènent à la découverte de nouveaux liens et de nouveaux nœuds. La figure 4 montre ce que Williams obtient à partir du terme anglais plants dans l'un des corpus qu'il a traités. Le réseau peut ensuite être présenté à un lexicographe ou à un terminologue qui devra interpréter les liens fournis. Selon Williams, un élément lexical partageant de très nombreux liens collocationnels peut révéler sa centralité dans le domaine. Par ailleurs, différents indices peuvent révéler les thématiques d'intérêt dans le domaine (les types de plantes, par exemple, si on se reporte à l'exemple de la figure 4). L'auteur soutient également que son approche devrait contribuer à revoir la manière traditionnelle d'élaborer des dictionnaires spécialisés puisque la combinatoire constitue une porte d'accès au sens. Circonscrire le sens d'un mot implique qu'on prenne en compte les unités avec lesquelles celui-ci se combine :

7. De manière générale, l'information mutuelle évalue la fréquence de la combinaison de deux éléments par rapport à leur fréquence d'apparition totale dans le corpus. Si la fréquence d'une combinaison spécifique est disproportionnée par rapport au nombre total d'occurrences des éléments, on estime qu'il s'agit d'une combinaison significative. 
meaning will cease to be solely associated with "words," but with the company the words keep, each associated frame of reference is representative of a particular conceptual network. (Williams, 1998, p. 162)

\subsection{La combinatoire comme soutien à l'analyse conceptuelle}

Ingrid Meyer et Kristen Mackintosh (1994) proposent également de faire appel à la combinatoire comme complément à l'analyse des concepts d'un domaine spécialisé. La méthode exploite le corpus spécialisé et montre que l'examen attentif des occurrences des termes et de leur combinatoire permet d'affiner et de formaliser partiellement l'analyse des concepts. Les auteurs examinent plus précisément des groupes appelés phrasèmes ${ }^{8}$ dont l'étude permet de découvrir ce qui suit (les exemples sont ceux des auteures) ${ }^{9}$ :

- Des phénomènes de multidimensionnalité (Bowker et Meyer, 1993), selon lesquels un concept peut être envisagé dans perspectives différentes: par exemple, erasable disk fait ressortir des caractéristiques relatives à l'écriture sur le support; bigh-capacity disk et low-capacity disk mettent en évidence la capacité de stockage du support.

- Des jeux de caractéristiques centrales d'un concept (que les auteures appellent conceptual frame) : par exemple, built-in $C D-R O M$ drive montre qu'il s'agit d'une composante périphérique de quelque chose; glass optical disk fait ressortir une composante du support.

- Distinguer des concepts entre eux: dans mastering facility, mastering désigne le procédé général qui consiste à convertir des données numériques sur des CD-ROM produits en bloc; dans desktop mastering, mastering designe un sous-procédé du procédé général (Meyer et Mackintosh, 1994).

À l'inverse, l'analyse conceptuelle permet de mieux comprendre la combinatoire (phrasèmes) et de la prédire jusqu'à un certain point. Les auteures font notamment appel à l'héritage, c'est-à-dire le fait pour un concept spécifique d'hériter des caractéristiques d'un

8. Les auteurs prennent en compte les termes complexes de nature nominale (compounds) et les collocations à proprement parler, confirmant le problème de distinction entre ces deux groupes signalés dans la section précédente.

9. Dans un article écrit en 1996 (Meyer et Mackintosh, 1996), les auteures donnent une liste plus riche des enseignements qu'un terminologue peut tirer de l'analyse des phrasèmes. 
générique, pour expliquer des combinaisons verbales et leur régime. Cette idée rejoint le constat évoqué à la section 3 selon lequel un même collocatif peut se combiner avec des ensembles de termes.

La méthode de Meyer et Mackintosh est conçue pour permettre aux terminologues de parfaire l'analyse des concepts en vue d'une implémentation dans des bases de connaissances terminologiques. Elle pourrait contribuer à l'enrichissement des banques de terminologie plus classiques. Elle a le mérite de prendre en compte les occurrences des termes désignant les concepts en corpus spécialisé et ainsi de mettre au jour des propriétés linguistiques (comme la polysémie, le régime verbal, etc.). Elle montre que l'analyse de l'environnement immédiat du terme permet un meilleur accès aux connaissances spécialisées. La démarche de Meyer et Mackintosh reste essentiellement conceptuelle tant dans les objectifs que dans le déploiement de la méthode. La combinatoire y est envisagée comme un élément parmi d'autres à prendre en compte dans l'acquisition des connaissances et reste utile dans la mesure où elle permet une définition plus précise des concepts. La proposition de Meyer et Mackintosh - et jusqu'à un certain point celle de Williams (1998) repose donc sur l'hypothèse selon laquelle concept et fonctionnement linguistique se rejoignent, et que deux plans descriptifs sont non seulement compatibles, mais se complètent l'un l'autre.

\section{La combinatoire : perspective lexicographique}

J'ai évoqué à la section 3 quelques travaux proposant des stratégies pour prendre en compte la combinatoire des termes dans les ressources terminologiques. Certaines stratégies étaient plus élaborées que d'autres, mais aucune n'imposait une refonte de la structure de base des fiches ou des entrées terminologiques. Dans tous les cas, il s'agissait d'ajouter de l'information à des entrées consacrées à des termes de nature nominale.

Dans la présente section, je décrirai des travaux qui vont un peu plus loin dans l'encodage des combinaisons dans lesquelles apparaissent les termes. Il ne s'agit plus ici d'ajouter des collocatifs au contenu de ressources terminologiques existantes, mais plutôt d'envisager des solutions d'encodage conçues dès le départ pour accueillir des éléments caractérisant la combinatoire des termes. Nous verrons également que certains de ces travaux abordent les notions de terme et de collocation en adoptant une perspective résolument lexicographique. 


\subsection{Trois dictionnaires décrivant la combinatoire spécialisée}

Dans un lexique portant sur un ensemble de termes du domaine de la bourse, Betty Cohen (1986) organise les cooccurrents (terme choisi par l'auteure) selon deux paramètres : en abscisse, les cooccurrents sont répartis en fonction de leur partie du discours (deux colonnes sont consacrées aux cooccurrents verbaux et distinguent la fonction du terme : sujet ou objet); en ordonnée, de grandes classes sémantiques correspondant à différents stades du cycle économique servent à affiner le classement (DÉBUT, CROISSANCE, DÉCLIN, FIN) (figure 5).

INDUSTRIE : Ensemble des activités, des métiers d'un pays ou d'un secteur qui produisent des richesses pour la mise en œuvre des matières premières.

\begin{tabular}{|c|c|c|c|c|}
\hline & NOMS & $\begin{array}{l}\text { VERBES } \\
\text { (SUJET) }\end{array}$ & $\begin{array}{l}\text { VERBES } \\
\text { (OBJETS) }\end{array}$ & ADJECTIFS \\
\hline DÉBUT & $\begin{array}{l}\text { redéploiement } \\
\text { redressement } \\
\text { relance }\end{array}$ & $\begin{array}{l}\text { naître } \\
\text { se redéployer } \\
\text { se redresser }\end{array}$ & $\begin{array}{l}\text { créer } \\
\text { redresser } \\
\text { relancer }\end{array}$ & \\
\hline CROISSANCE & $\begin{array}{l}\text { développement } \\
\text { essor } \\
\text { expansion } \\
\text { progrès }\end{array}$ & $\begin{array}{l}\text { croître } \\
\text { se développer } \\
\text { sétendre } \\
\text { progresser }\end{array}$ & $\begin{array}{l}\text { développer } \\
\text { stimuler }\end{array}$ & $\begin{array}{l}\text { croissante } \\
\text { en essor } \\
\text { en expansion } \\
\text { florissante } \\
\text { forte } \\
\text { grosse } \\
\text { importante } \\
\text { imposante } \\
\text { productive } \\
\text { prospère }\end{array}$ \\
\hline INDÉTERMINÉS & & stagner & & $\begin{array}{l}\text { stable } \\
\text { stagnante }\end{array}$ \\
\hline DÉCLIN & $\begin{array}{l}\text { défaillance } \\
\text { (dans) }\end{array}$ & $\begin{array}{l}\text { défaillir } \\
\text { péricliter }\end{array}$ & & $\begin{array}{l}\text { défaillante } \\
\text { déprimée } \\
\text { faible } \\
\text { maigre } \\
\text { périclitante } \\
\text { petite }\end{array}$ \\
\hline \multicolumn{5}{|l|}{ FIN } \\
\hline $\begin{array}{l}\text { AUTRES } \\
\text { COOCCURRENTS }\end{array}$ & $\begin{array}{l}\text { concentration } \\
\text { groupement } \\
\text { intégration } \\
\text { privatisation } \\
\text { étatisation } \\
\text { nationalisation } \\
\text { socialisation }\end{array}$ & & $\begin{array}{l}\text { avoir } \\
\text { posséder } \\
\text { privatiser } \\
\text { étatiser } \\
\text { nationaliser }\end{array}$ & $\begin{array}{l}\text { concentrée } \\
\text { dispersée } \\
\text { privée } \\
\text { collectivisée } \\
\text { d'État } \\
\text { nationalisée } \\
\text { socialisée }\end{array}$ \\
\hline
\end{tabular}

Figure 5. Entrée industrie et cooccurrents (Cohen, 1986, p. 89) 
Ces classes sémantiques s'appuient sur le système des fonctions lexicales (Mel'čuk, 1996) sur lequel je reviendrai plus loin. Un classement sémantique de cooccurrents est également proposé par Isabelle Laporte (1996) et Miriam Castro Buendía (2013). Même si ces dernières auteures ne font pas appel aux fonctions lexicales à proprement parler, le principe général selon lequel on peut organiser les collocatifs en fonction de leur sens est appliqué.

Dans un dictionnaire portant sur le français des affaires, Jean Binon et al. (2000) ont conçu pour certains articles une structure très développée qui comprend notamment des rubriques consacrées aux familles de termes (termes apparentés morphologiquement), aux renvois analogiques, aux définitions, aux exemples, et des rubriques consacrées aux expressions multilexémiques parmi lesquelles figurent les collocations. À la figure 6, j’ai reproduit une partie de la liste des collocatifs verbaux répertoriés pour le terme produit.

\section{un PRODUIT [...]}

1.1 Bien ou service réalisé par un agent économique (un agriculteur, un artisan, une entreprise, un État - X) et qui est destiné à la satisfaction des besoins des autres agents économiques $(\mathrm{Y})$.

+ verbe : qui fait quoi?

\section{$\mathrm{X}$ (une entreprise)}

$\mathrm{X}$ (une entreprise)

$\mathrm{X}$ (une entreprise)

$\mathrm{X}$ (une entreprise)

$\mathrm{X}$ (une entreprise)

$\rightarrow$ un (nouveau)

\section{$[\ldots]$}

Y (un consommateur, demander un une entreprise) concevoir un

développer un

mettre au point un

fabriquer un

lancer un nouveau $\sim$ sur le marché

commercialiser un

mettre en vente un

sortir un nouveau $\sim$ sur le marché

apparaître sur le marché sortir

commander un à X acheter un

consommer un la conception d'un le développement d'un la mise au point d'un la fabrication d'un le lancement d'un

la commercialisattion d'un la mise en vente d'un la sortie d'un

l'apparition d'un (sur le marché) la sortie d'un

la demande de $\sim /$ pour un la commande d'un l'achat d'un la consommation d'un

Figure 6. Partie de l'article produit (Binon et al., 2000, p. 443-446) 
Binon et al. $(2000)^{10}$ ont adapté et simplifié un système conçu pour décrire les collocations (et plus généralement les liens lexicaux) de langue commune, comme l'avait fait Cohen (1986) avant eux, à savoir les fonctions lexicales (Mel'čuk, 1996).

Les fonctions lexicales sont également utilisées pour décrire les collocations dans deux dictionnaires spécialisés en ligne, l'un consacré à l'informatique (DiCoInfo, 2018), l'autre à l'environnement (DiCoEnviro, 2018). Comme le dictionnaire de Binon et al., le DiCoInfo et le DiCoEnviro comportent d'autres rubriques (structure actancielle, définition, liens paradigmatiques - antonymie, synonymie, etc. - contextes). La figure 7 (page suivante) montre comment les collocatifs verbaux du terme Internet (ainsi que les nominalisations de ces verbes) sont décrits dans le DiCoInfo ${ }^{11}$. Un peu à la manière du Cohen (1986), les collocatifs sont regroupés par classes (selon un système décrit dans L'Homme et al., 2012). En outre, le sens des collocatifs est décrit au moyen d'une courte explication. Enfin, comme certains collocatifs font eux-mêmes l'objet d'un article (par exemple, naviguer, rechercher, se connecter), un hyperlien permet d'accéder à cet article directement.

On peut se demander à ce stade pourquoi les trois exemples choisis s'adossent au système des fonctions lexicales (Mel'čuk, 1996) pour décrire la combinatoire spécialisée. À l'origine, les fonctions lexicales ont été développées pour rendre compte de la combinatoire de manière générale et non comme un outil spécifique de description de la combinatoire des termes. Or, il semble que les lexicographes ou terminologues à l'origine des dictionnaires cités en exemples dans la présente section y ont vu un système permettant une description bonifiée des collocations relevant de quatre domaines distincts (affaires, bourse, environnement et informatique). À la section suivante, je donne une brève description des fonctions lexicales et montre comment elles ont été utilisées dans les dictionnaires choisis pour organiser les collocations et les présenter.

10. Une version électronique de ce dictionnaire a également été mise à disposition pendant une certaine période, offrant des accès et des modes de présentation plus souples que ceux offerts par le dictionnaire papier.

11. Comme il s'agit d'un dictionnaire en ligne, la reproduction des collocations est simplifiée ici. Dans la version originale, un système d'onglets et de rubans permet aux utilisateurs de naviguer dans les liens de combinatoire en fonction des classes auxquelles ils appartiennent. 
Internet, n. m.

Réseau mondial, constitué d'un ensemble complexe de réseaux plus petits pouvant communiquer entre eux grâce des protocoles et des interfaces normalisés, utilisé par des utilisateurs ๑) pour consulter des sites $\odot$ ou utiliser des ressources $\odot$, généralement au moyen d'un navigateur.

\begin{tabular}{|c|c|}
\hline \multicolumn{2}{|l|}{ Mettre quelque part } \\
\hline \multirow[t]{2}{*}{ Qqn ou qqch. met le site ou la ressource dans I. } & $\begin{array}{l}\text { mettre ... en ligne } \\
\text { publier }{ }_{1} \ldots \text { dans } ~ \\
\text { diffuser } \ldots \text { dans } \sim\end{array}$ \\
\hline & $\begin{array}{l}\text { publication }_{1} \text { de } \ldots \text { dans } \sim \\
\text { mise en ligne de ... } \\
\text { diffusion de } . . . \text { dans } \sim\end{array}$ \\
\hline \multicolumn{2}{|l|}{ Utiliser / Ne pas utiliser } \\
\hline \multicolumn{2}{|l|}{ Commencer à utiliser / Apparaître } \\
\hline \multirow[t]{2}{*}{ L'utilisateur commence à utiliser I. } & $\begin{array}{l}\text { se connecter }{ }_{1} \text { à } ~ \\
\text { accéder }{ }_{2} \text { à } ~ \\
\text { aller dans } \sim\end{array}$ \\
\hline & $\begin{array}{l}\text { connexion }_{1.1} \text { à } \\
\text { accès }_{2 \mathrm{a} a} \text { à }\end{array}$ \\
\hline \multicolumn{2}{|l|}{ Utiliser / Faire fonctionner } \\
\hline L'utilisateur utilise I. & naviguer $_{1}$ dans \\
\hline \multirow[t]{2}{*}{ La ressource ou le site est dans I. } & $\begin{array}{l}\text { circuler }{ }_{1 \frac{1}{1}} \text { dans } ~ \\
\text { etre en ligne }\end{array}$ \\
\hline & circulation $_{1}$ dans $\sim$ \\
\hline \multirow[t]{2}{*}{$\begin{array}{l}\text { L'utilisateur utilise I. pour intervenir sur la } \\
\text { ressource ou le site }\end{array}$} & $\begin{array}{l}\text { rechercher }{ }_{1} \ldots \text { dans } \sim \\
\operatorname{chercher}_{1} \ldots \text { dans } \sim\end{array}$ \\
\hline & recherche $_{1}$ de $\ldots$ dans $~$ \\
\hline $\begin{array}{l}\text { L'utilisateur utilise I. pour intervenir sur la } \\
\text { ressource ou le site }\end{array}$ & trouver ... dans $\sim$ \\
\hline \multicolumn{2}{|l|}{ Cesser d'utiliser / De faire fonctionner } \\
\hline \multirow[t]{2}{*}{ L'utilisateur cesse d'utiliser I. } & se déconnecter ${ }_{1} \mathrm{~d}^{\prime} \sim$ \\
\hline & déconnexion $_{1_{-}^{-}} \mathrm{d}^{\prime} \sim$ \\
\hline
\end{tabular}

Figure 7. Partie de l'article Internet (DiCoInfo, 2018)

\subsection{Les fonctions lexicales : application à la terminologie}

Les fonctions lexicales permettent de décrire trois propriétés des collocations : leur structure syntaxique, leur sens (général et abstrait) et le lien de la collocation avec la structure actancielle de la base (ou mot clé). Voyons comment ces trois propriétés sont prises en compte dans les dictionnaires cités en exemples dans la section 5.1. 
1) En ce qui concerne la structure syntaxique, on remarque que le lien entre la base et le collocatif n'est pas toujours le même d'une collocation à l'autre :

Base + verbe $(+\ldots)$

une industrie se redresse, une industrie croît (Cohen, 1986, p. 69)

un produit sort, un produit apparaît sur ... (Binon et al., 2000, p. 445)

un cyclone dévaste ..., une menace pèse sur ... (DiCoEnviro, 2018, n.p.)

un virus infecte ... (DiCoInfo, 2018, n.p.)

Verbe + base

créer une industrie, développer une industrie (Cohen, 1986, p. 69)

développer un produit, consommer un produit (Binon et al., 2000, p. 445)

constituer une menace, produire de l'électricité (DiCoEnviro, 2018, n.p.) taper au clavier, ouvrir une fenêtre, créer un mot de passe (DiCoInfo, 2018, n.p.)

Verbe $+\ldots+$ base

introduire ... dans un habitat, recharger ... à une borne (DiCoEnviro, 2018, n.p.)

entrer ... au clavier, accéder à ... avec un mot de passe, chercher ... dans Internet (DiCoInfo, 2018, n.p.).

L'information donnée sur la structure syntaxique des collocations reste minimale dans le lexique de Cohen (1986). Outre la création de deux colonnes distinctes consacrées aux collocations verbales dans lesquelles le terme est sujet et aux collocations dans lesquelles le terme est objet, la structure syntaxique doit être inférée par l'utilisateur. L'information relative à cette première propriété est beaucoup plus explicite dans Binon et al. (2000) et dans le DiCoInfo (2018). Le collocatif est présenté, et le terme est remplacé par un $\sim$ montrant sa position par rapport au collocatif : consommer un (produit) (Binon et al., 2000, p. 445); chercher ... dans (Internet) (DiCoInfo, 2018, n.p.). La présentation de la structure syntaxique des collocations permet à un utilisateur d'avoir immédiatement accès à la manière de les construire dans les textes.

2) En ce qui concerne le sens, on remarque que des collocatifs différents se distribuent dans des classes sémantiques plus grandes: 
«créer»

créer une industrie (Cohen, 1986, p. 69)

développer un produit (Binon et al., 2000, p. 445)

créer un mot de passe (DiCoInfo, 2018, n.p.)

produire de l'électricité (DiCoEnviro, 2018, n.p.)

«Se trouver quelque part»

un produit sort, un produit apparaît sur ... (Binon et al., 2000, p. 445)

«Agir sur quelque chose»

un cyclone dévaste ... une menace pèse sur ... (DiCoEnviro, 2018, n.p.)

un virus infecte ... (DiCoInfo, 2018, n.p.)

«Utiliser»

introduire ... dans un habitat, recharger ... à une borne (DiCoEnviro, 2018, n.p.)

consommer un produit (Binon et al., 2000, p. 445)

taper au clavier, ouvrir une fenêtre, entrer ... au clavier, accéder à ...

avec un mot de passe, chercher ... dans Internet (DiCoInfo, 2018, n.p.)

«être»

constituer une menace (DiCoEnviro, 2018, n.p.)

La prise en compte du sens des collocatifs permet aux terminologues d'aller au-delà du simple classement en parties du discours. Ce sens est expliqué au moyen de paraphrases simplifiées dans le DiCoEnviro (2018) et le DiCoInfo (2018), donnant à l'utilisateur un complément qu'une simple énumération ne peut pas fournir. Il peut également être à la base d'un regroupement des collocatifs en classes plus générales, comme dans Cohen (1986) et le DiCoInfo (2018). Ce regroupement en classes permet de rapprocher des collocatifs de sens voisins ou de mieux mettre en évidence des distinctions (comme le DÉBUT et la FIN dans Cohen (1986) ou le «Commencer à utiliser» et le «Cesser d'utiliser» du DiCoInfo (2018).

3) Enfin, en ce qui concerne le lien avec la structure actancielle de la base, on constate que les collocations font appel de manière différente aux actants. L'établissement du lien avec la structure actancielle permet de distinguer les collocatifs en fonction de l'actant (ou des actants) auquel ils font appel. Par exemple, on remarque dans l'entrée produit (Binon et al., 2000, p. 445) que "consommer un produit» fait appel au deuxième actant de produit (représenté par 
$\mathrm{Y})$ et que «développer un produit» fait appel au premier actant $(\mathrm{X})$. Les actants eux-mêmes sont explicités dans la définition (figure 6). De même, on remarque dans l'entrée consacrée à Internet extraite du DiCoInfo (2018, n.p.) que «naviguer dans Internet» fait appel au premier actant (utilisateur) alors que «rechercher ... dans Internet» fait appel aux deux actants (utilisateur, mais également site et ressource) (figure 7). On constate que les explications mettent en évidence les liens entre les collocations et la structure actancielle en rappelant les étiquettes des actants (ex. Lutilisateur utilise I. ou Lutilisateur utilise I. pour intervenir sur le site ou la ressource). La même observation peut être faite au sujet des collocations dans lesquelles apparaît le terme menace (DiCoEnviro, 2018, n.p.). En considérant la structure actancielle suivante pour menace: $\mathrm{X}$ (changement ou activité) est une $\sim$ pour $Y$ (écosystème, espèce), on remarque que «constituer une menace» fait appel à $\mathrm{X}$ ( «un changement ou une activité constituent une menace») alors que une peser fait appel à $Y$ ( «une menace pèse sur un écosystème ou une espèce $»)^{12}$.

En réfléchissant à l'encodage des collocations en amont, les dictionnaires dont il vient d'être question évitent les écueils évoqués à la section 3 concernant l'ajout des collocatifs à des ressources terminologiques existantes. Certains choix méthodologiques contournent à la fois la question du mélange de deux plans descriptifs (le conceptuel et le linguistique) et le problème de la distinction entre termes complexes et collocations (les trois dictionnaires décrits ici traitent principalement les termes simples). Par ailleurs, une description riche de la combinatoire spécialisée au moyen d'un système comme celui des fonctions lexicales permet d'aller beaucoup plus loin dans la manière d'encoder les collocations et de les présenter à l'utilisateur que les solutions décrites à la section 3. Chacune des propriétés que nous avons évoquées peut donner lieu à un nouveau type de

12. Ces trois propriétés (structure syntaxique de la collocation, sens général et lien avec la structure actancielle du mot clé) sont représentées formellement dans une fonction lexicale. Par exemple, il existe un trio de fonctions lexicales qui représente la réalisation inhérente au sens du mot clé $\left(\right.$ Fact $_{\mathrm{i}}$, Real $_{\mathrm{i}}$, Labreal $\left._{\mathrm{ij}}\right)$ : la première s'utilise lorsque le mot clé est sujet, la seconde et la troisième s'emploient lorsque le mot clé est $1^{\text {er }}$ ou $2^{\text {e }}$ complément respectivement. L'indice explicite le lien avec le mot clé. On obtiendra ainsi : Fact $_{2}($ menace $)=\sim$ pèse sur ...; Fact $_{2}($ virus $)=$ infecte ...; $\operatorname{Real}_{1}($ clavier $)=$ taper au $\sim \operatorname{Real}_{1}($ fenêtre $)=$ ouvrir une $\sim \operatorname{Real}_{2}($ produit $)=$ consommer un $\sim$ Labreal $_{12}($ Internet $)=$ chercher ... dans $\sim ;$ Labreal $_{12}($ clavier $)=$ entrer ... au . Compte tenu du manque de transparence des fonctions lexicales, différents dispositifs ont été proposés par les lexicographes et terminologues pour simplifier leur présentation dans les ressources (voir notamment Mel'čuk et Polguère, 2007). 
classement. Cependant, l'application de telles méthodes soulève quelques questions quant au travail que doit réaliser le terminologue. On peut certes saluer le fait qu'elles mettent en évidence les propriétés linguistiques des deux éléments entrant dans la formation d'une collocation spécialisée, à savoir le terme et son collocatif. Cependant, cette mise en évidence se fait au moyen d'un système complexe dont on voit mal l'introduction harmonieuse dans des ressources terminologiques de facture plus classique. Enfin, les choix méthodologiques ont également comme conséquence de mettre à l'écart les considérations visant à ancrer les descriptions de termes dans une représentation des connaissances d'un domaine, puisqu'on se focalise sur le linguistique.

\section{Un bilan pour la terminologie}

Les travaux sur la combinatoire des termes que j'ai présentés succinctement dans les sections qui précèdent ont grandement contribué à faire en sorte que les terminologues, lorsqu'ils abordent un corpus spécialisé, y recherchent autre chose que des attestations de termes et des indices des concepts que ces termes dénotent (sous la forme de définitions, de contextes définitoires ou de contextes explicatifs). Évidemment, ces travaux s'inscrivent dans un contexte où le corpus de manière générale occupe une large place dans les analyses terminologiques. Il faut donc aborder l'apport des travaux portant sur la combinatoire dans ce plus large contexte. Force est de constater que ces travaux ont contribué à étendre l'analyse terminologique à l'environnement linguistique immédiat dans lequel évoluent les termes. On ne remet plus en question l'intérêt que présente la combinatoire spécialisée dans les cercles terminologiques, et cela, indépendamment des perspectives adoptées.

On ne peut toutefois que déplorer qu'il n'y ait pas davantage de ressources terminologiques tenant compte de la combinatoire des termes. Il est vrai que même si on s'entend sur l'intérêt de décrire cette combinatoire, certaines ressources terminologiques peuvent difficilement accueillir ce type d'information. Nous avons évoqué quelques difficultés entraînées par ces ajouts dans les banques de terminologie; on peut supposer qu'elles prendront une importance accrue dans les thésaurus, les bases de connaissances terminologiques et les ontologies dont la structure même repose sur l'établissement de liens formels et explicites entre concepts (et non entre termes à proprement parler). 
Au-delà de l'enrichissement des ressources terminologiques, la prise en compte effective de l'environnement linguistique des termes a eu un impact beaucoup plus profond sur le travail terminologique. Pour les uns, elle permet de délimiter les concepts qui échappent à l'observation directe; pour les autres, elle constitue une manière d'appréhender leur sens. Ainsi, l'analyse des concepts préconisée par l'approche terminologique plus classique qui consiste à définir les concepts en fonction de leur place dans une structure de connaissances - en occultant les propriétés linguistiques des termes - se trouve enrichie d'une dimension et devient plus accessible au terminologue.

L'analyse de la combinatoire spécialisée a permis à la terminologie d'approfondir ou de mieux circonscrire certaines propriétés conceptuelles des termes: leur multidimensionnalité, évoquée par Meyer et Mackintosh (1994); la centralité des concepts (Williams, 1998); les caractéristiques rattachées à un concept ou à des concepts spécifiques (Meyer et Mackintosh, 1994; Williams, 1998); la combinaison de collocatifs avec des classes de termes (Heid, 1994), etc. Toutefois, on peut se demander si le fonctionnement linguistique des termes constitue un reflet fidèle de l'appartenance des concepts qu'ils dénotent à un système conceptuel. On pourrait s'attendre à ce qu'une analyse intégrant la combinatoire parvienne à des résultats qui ne soient pas entièrement compatibles avec ceux livrés par une analyse qui n'en tient pas compte. Autrement dit, les représentations issues de l'une ou l'autre analyse sont susceptibles de différer.

L'analyse de la combinatoire spécialisée a également permis de mettre en lumière différents phénomènes qui échappaient auparavant plus facilement à l'observation. D'abord, ce sont probablement ces travaux qui ont amené les terminologues à se pencher sur tout un ensemble d'unités linguistiques qui font désormais partie intégrante de l'analyse terminologique. On peut évoquer le cas des unités lexicales appartenant à des parties du discours autres que le nom, le verbe notamment. Pour les uns, les unités verbales sont définies comme étant périphériques au terme de nature nominale: elles servent à mieux appréhender un concept ou des concepts voisins. Pour les autres, certains verbes peuvent prétendre au statut d'unité terminologique. Malgré ces différences de perspectives, force est de constater que les terminologues s'intéressent d'une manière ou d'une autre à la contribution du verbe à l'expression de connaissances spécialisées; le verbe a fait l'objet de multiples caractérisations au 
cours des vingt dernières années (L'Homme, 1998; Lerat, 2002; Lorente, 2007, entre autres).

Malgré le chemin parcouru depuis les premiers travaux portant sur la combinatoire spécialisée, certaines questions centrales restent à explorer. La première est celle de la distinction entre terme complexe et collocation. François Maniez (2002) avait proposé de faire reposer cette distinction - en ce qui concerne les groupes composés d'un nom et d'un adjectif - sur la nature de l'adjectif lui-même. Selon lui, les groupes dans lesquels l'adjectif est relationnel sont plus susceptibles de correspondre à des termes complexes alors que ceux dans lesquels l'adjectif est qualificatif correspondraient à des collocations. Ceci permettrait de définir hépatite virale comme un terme complexe et hépatite aiguë comme une collocation. Nous avons ont mis en évidence les difficultés que soulève cette distinction (L'Homme et Jia, 2015).

L'analyse de la combinatoire spécialisée donne également lieu à de nouvelles pistes. Mocja Pecman (2012), par exemple, propose de tenir compte d'un nouveau paramètre dans l'observation de la combinatoire en corpus spécialisé, à savoir sa contribution au discours spécialisé. Selon l'auteure, une partie des collocations présentes dans les corpus spécialisés sont associées à des discours (genres textuels ou mouvements rhétoriques) et transcendent les frontières entre domaines, alors que d'autres seraient plutôt associées à des disciplines.

Par ailleurs, la combinatoire est un phénomène lexical au sens large qui a d'abord intéressé les lexicologues et lexicographes. Or, peu de travaux ont exploré les intersections inévitables entre les collocations décrites par les lexicographes et les collocations spécialisées. On pourrait sans doute s'attendre à des recoupements, mais également à des divergences. Enfin, les analyses interlinguistiques pourraient donner lieu à de nouvelles pistes également. Les structures linguistiques différant d'une langue à l'autre, il est permis de supposer qu'elles affecteront le fonctionnement des collocations.

\section{Références}

Béjoint, Henri et Philippe Thoiron (1989). «Pour un index cumulatif et évolutif de cooccurrents en langue techno-scientifique sectorielle». Meta, 34, 4, p. 661-671.

Benson, Morton, Evelyne Benson et Robert Ilson (1986). The BBI Combinatory Dictionary of English: A Guide to Word Combinations. Amsterdam/Philadelphie, John Benjamins. 
Binon, Jean, Serge Verlinde, Jan van Dyck et Ann Bertels (2000). Dictionnaire d'apprentissage du français des affaires. Paris, Didier.

Bowker, Lynne et Ingrid Meyer (1993). "Beyond "Textbook" Concept Systems: Handling Multidimensionality in a New Generation of Term Banks». In K. D. Schmitz, dir. Proceedings of Terminology and Knowledge Engineering (TKE'93). Frankfurt, Indeks 30 Verlag, p. 123-137.

Buendía Castro, Miriam (2013). Phraseology in Specialized Language and its Representation in Environmental Knowledge Resources. Thèse de doctorat, Departamento de traducción e interpretación, Universidad de Granada. Inédit.

Caignon, Philippe (2001). Vocabulaire de cooccurrents de la comptabilité. Brossard (Québec), Linguatech.

Church, Ken et Patrick Hanks (1990). «Word Associations, Norms, Mutual Information, and Lexicography». Computational Linguistics, 16, 1, p. 2229.

Clas, André (1984). «Collocations et langues de spécialité». Meta, 39, 4, p. 576-580.

Cohen, Betty (1986). Lexique de cooccurrents : Bourse - conjuncture. Brossard (Québec), Linguatech.

DiCoEnviro. Dictionnaire fondamental de l'environnement (2018). Disponible à <http://olst.ling.umontreal.ca/cgi-bin/dicoenviro/search_enviro.cgi> [consulté le 3 janvier 2018].

DiCoInfo. Dictionnaire fondamental de l'informatique et de l'Internet (2018). Disponible à <http://olst.ling.umontreal.ca/cgi-bin/dicoinfo/search. cgi> [consulté le 3 janvier 2018].

Hausmann, Franz Joseph (1979). «Un dictionnaire des collocations est-il possible?». Travaux de Linguistique et de Littérature, 17, 1, p. 187-195.

Heid, Ulrich (1994). "On the Way Words Work Together. Topics in Lexical Combinatorics». In W. Martin et al., dir. Euralex 94 Proceedings. Amsterdam, Vrije Universiteit, p. 226-257.

Heid, Ulrich et Gerhard Freibott (1991). «Collocations dans une base de données terminologique et lexicale». Meta, 36, 1, p. 77-91.

Kilgarriff, Adam et David Tugwell (2001). «WORD SKETCH: Extraction, Combination and Display of Significant Collocations for Lexicography». In Proceedings of the Workshop on Collocations: Computational Extraction, Analysis and Exploitation, ACL-EACL 2001. Toulouse, p. 32-38.

Lainé, Claude (1993). Vocabulaire combinatoire de la CFAO mécanique. Ottawa, Secrétariat d'État du Canada/Réseau international de néologie et de terminologie.

Larivière, Louise (1998). «Valeur sémantique du verbe dans les collocations spécialisées». TTR, 11, 1, p. 173-197.

Laporte, Isabelle (1996). Méthode de recensement et de présentation des combinaisons lexicales en langue de spécialité, accompagnée d'un lexique 
combinatoire de la pharmacologie cardiovasculaire. Travail dirigé, Département de linguistique et de traduction, Université de Montréal. Inédit.

Lerat, Pierre (2002). «Qu'est-ce que le verbe spécialisé? Le cas du droit». Cahiers de Lexicologie, 80, p. 201-211.

L'Homme, Marie-Claude (1997). «Organisation des classes conceptuelles pour l'accès informatisé aux combinaisons lexicales spécialisées verbe + nom». In Actes des deuxièmes rencontres Terminologie et intelligence artificielle, TIA-97. Toulouse, Université Toulouse-le-Mirail, p.161174.

L'Homme, Marie-Claude (1998). «Définition du statut du verbe en langue de spécialité et sa description lexicographique». Cahiers de lexicologie, 73, p. 61-84.

L'Homme, Marie-Claude (2000). «Understanding Specialized Lexical Combinations». Terminology, 6, 1, p. 89-110.

L'Homme, Marie-Claude et Zhichao Jia (2015). "Classement des combinaisons lexicales spécialisées à base nominale dans un dictionnaire d'informatique». Cabiers de lexicologie, 106, p. 229-251.

L'Homme, Marie-Claude, Benoît Robichaud et Patrick Leroyer (2012). «Encoding Collocations in DiCoInfo: From Formal to Userfriendly Representations». In S. Granger et M. Paquot, dir. Electronic Lexicography. Oxford, Oxford University Press, p. 211-236.

Lorente,Mercè (2007). «Les unitats lèxiques verbals dels textos especialitzats. Redefinició d'una proposta de classificació». In M. Lorente et al., dir. Estudis de lingüistics $i$ de lingüistica aplicada en honor de M. Teresa Cabré Catellvi. Volum II: De deixebles. Barcelona, Institut Universitari de Lingüística Aplicada de la Universitat Pompeu Fabra, Sèrie Monografies 11-12, p. 365-380.

Maniez, François (2002). «Distinguer les termes des collocations : étude sur corpus du patron < Adjectif - Nom> en anglais médical». In Actes de la conférence Traitement automatique de la langue naturelle - TALN (2002). Nancy, p. 345-350.

Martin, Willy (1992). «Remarks on Collocations in Sublanguages». Terminologie et traduction, 2-3, p. 157-164.

Mel'čuk, Igor (1996). «Lexical Functions: A Tool for the Description of Lexical Relations in the Lexicon». In L. Wanner, dir. Lexical Functions in Lexicography and Natural Language Processing. Amsterdam/ Philadelphie, John Benjamins, p. 37-102.

Mel'čuk, Igor et Alain Polguère (2007). Lexique actif du français. L'apprentissage du vocabulaire fondé sur 20000 dérivations sémantiques et collocations du français. Bruxelles, De Boeck.

Meyer, Ingrid et Kristen Mackintosh (1994). «Phraseme Analysis and Concept Analysis: Exploring a Symbiotic Relationship in the Specia- 
lized Lexicon». In W. Martin et al., dir. Euralex 94 Proceedings. Amsterdam, Vrije Universiteit, p. 338-348.

Meyer,Ingrid et Kristen Mackintosh (1996). «Refining the Terminographer's Concept-analysis Methods: How can Phraseology Help?». Terminology 3, 2, p. 1-26.

Meynard, Isabelle (1998). «Combinaisons lexicales en langue de spécialité : problèmes et perspectives». In Z. Guével et E. Valentine, dir. Traduction et langues de spécialité: approches théoriques et considérations pédagogiques. Québec, CIRAL, p. 123-138.

Oxford Online Collocation Dictionary (2018). Disponible à <http://www. freecollocation.com/> [consulté le 5 janvier 2018].

Pecman, Mocja (2012). «Étude lexicographique et discursive des collocations en vue de leur intégration dans une base de données terminologiques». JoSTrans. The Journal of Specialised Translation, 18. Disponible à <http:// www.jostrans.org/issue18/art_pecman.php> [consulté le 5 janvier 2018].

Pavel, Silvia (1993). «La phraséologie en langue de spécialité. Méthodologie de consignation dans les vocabulaires terminologiques». Terminologies nouvelles, 10, p. 23-35.

Picht, Heribert (1987). «Terms and their LSP Environment: LSP Phraseology». Meta, 32, 2, p. 149-155.

Sinclair, John M. (1991). Corpus, Concordance, Collocation. Oxford, Oxford University Press.

Smadja, Franz (1993). «Retrieving Collocations from Text: Xtract». Computational Linguistics, 19, 1, p. 143-177.

Termium Plus ${ }^{\circledR}$ (s.d.). Disponible à <http://www.btb.termiumplus.gc.ca/ tpv2alpha/alpha-fra.html?lang=fra $>$ [consulté le 3 janvier 2018].

Williams, Geoffrey (1998). «Collocational Networks: Interlocking Patterns of Lexis in a Corpus of Plant Biology Research Articles». International Journal of Corpus Linguistics, 3, 1, p. 151-171.

Marie-Claude L'Homme Observatoire de linguistique Sens-Texte (OLST) Département de linguistique et de traduction

Université de Montréal Montréal (Québec), Canada mc.lhomme@umontreal.ca 